\title{
Understanding Individual Defects in CdTe Solar Cells: From Atomic Structure to Electrical Activity
}

\author{
Chen $\mathrm{Li}^{1,2}$, Yelong $\mathrm{Wu}^{3}$, Jonathan Poplawsky ${ }^{1,4}$, Naba Paudel ${ }^{3}$, Timothy J. Pennycook ${ }^{5,6}$, Sarah J. \\ Haigh $^{7}$, Andrew R. Lupini ${ }^{1}$, Mark P. Oxley ${ }^{1}$, Yanfa Yan $^{3}$ and Stephen J. Pennycook ${ }^{4}$ \\ ${ }^{1}$ Materials Science and Technology Division, Oak Ridge National Laboratory, Oak Ridge, TN, USA \\ 2 Department of Chemistry, Vanderbilt University, Nashville, TN, USA \\ ${ }^{3}$ Department of Physics and Astronomy, The University of Toledo, Toledo, OH, USA \\ ${ }^{4}$ Department of Materials Science and Engineering, University of Tennessee, Knoxville, TN, USA \\ ${ }^{5}$ Department of Materials, University of Oxford, Oxford, OX13PH, UK \\ ${ }^{6}$ SuperSTEM Laboratory, Daresbury, WA44AD, UK \\ ${ }^{7}$ School of Materials, University of Manchester, Manchester, M139PL, UK
}

As one of the most promising thin film solar cell materials, CdTe has achieved the current record solar cell efficiency of $19.6 \%$ [1]. However there is still a considerable gap to its theoretical limit of $32 \%$. Defects such as dislocations, grain boundaries (GBs) and interfaces are usually considered to be major problems for high efficiency, as they are likely to be carrier recombination centers. However the photovoltaic behaviors of different defects are likely to be inhomogeneous, due to their diverse atomic configuration and bonding structure. Moreover, the defects may be passivated by chemical doping during the post-growth treatments. Particularly, after $\mathrm{CdCl}_{2}$ heat treatment, polycrystalline CdTe cells exceed single crystal cells in efficiency, which indicates that the GBs can play a positive role on the photovoltaic properties. In order to understand the electrical activity of individual defects, direct correlation from atomic and chemical structure to electronic property is critical.

Analytical aberration-corrected scanning transmission electron microscopes (STEM) equipped with electron energy loss spectroscopy (EELS) or energy dispersive X-ray (EDX) have been employed to resolve both the atomic structure and elemental distribution. Combining with electron-beam-induced current (EBIC) and density-functional theory (DFT), a structure-property correlation has been achieved on several types of defects. The EBIC map in Fig. 1(a) reveals that after $\mathrm{CdCl}_{2}$ treatment, the efficiency of GBs has been significantly enhanced. The STEM Z-contrast image in Fig. 1(b) shows periodic $\mathrm{Cd}_{3} \mathrm{Te}$ dislocation cores along a $\Sigma 9$ GB. The chemical distribution across the GB has been plotted with EELS in Fig. 1(c), showing that $\mathrm{Cl}$ has substituted for nearly half of the Te atoms within a confined range at the GB. The significant substitution of $\mathrm{Cl}$ for Te has been observed on basically all the GBs. DFT calculation confirms that the $\mathrm{Cl}$ segregation energy profile across the GB matches the narrow Cl EELS result. Moreover, it indicates that $\mathrm{Cl}$ doping removes the gap states and further dopes the GBs to n-type. This GB inversion creates p-n junctions between the grain interiors and the GBs (Fig. 1(d)), thereby separating electron-hole carriers into different conduction pathways and improving the efficiency.

Surprisingly, the intra-grain partial dislocation pairs (Fig. 2(a)) require no passivation from post-growth treatments. They do not create gap states but instead cause energy band bending (Fig. 2(b)), which assists the separation of electron-hole carriers, therefore reducing recombination [2, 3]. At the $\mathrm{CdTe} / \mathrm{CdS}$ interface, a significant Te-S interdiffusion has been found a few nanometers into the grain interiors (Fig. 3(a)). Meanwhile, long-range Te and S diffusion has also been detected along the GBs in CdS and CdTe, respectively. The Z-contrast image in Fig. 3(b) directly resolves $\mathrm{S}$ dopants in Te sites near the interface. A structure transformation from zinc-blend CdTe to wurtzite CdS has also been seen. STEM-EBIC indicates that the $\mathrm{p}-\mathrm{n}$ junction is $\sim 10 \mathrm{~nm}$ from the interface in CdTe, which is consistent with the $\mathrm{S}$ diffusion range in CdTe grains. The defects introduced by $\mathrm{Cu}$ treatment will also be discussed. [4] 


\section{References:}

[1] M. A. Green et al, Prog. Photovoltaics Res. Appl. 21, 827 (2013).

[2] C. Li et al, Ultramicroscopy 134, 113 (2013)

[3] C. Li et al, Phys. Rev. Lett. 111, 096403 (2013).

[4] This research was sponsored by the US DOE, Office of Energy Efficiency and Renewable Energy, Foundational Program to Advance Cell Efficiency (CL, YW, JP, NP, YY, SJP), and the Office of Basic Energy Sciences, Materials Science and Engineering Division (ARL, MPO). STEM-EDX research was supported by the UK National Facility for Aberration-Corrected STEM (TJP, SH). Computational facilities were provided by the National Energy Research Scientific Computing Center.

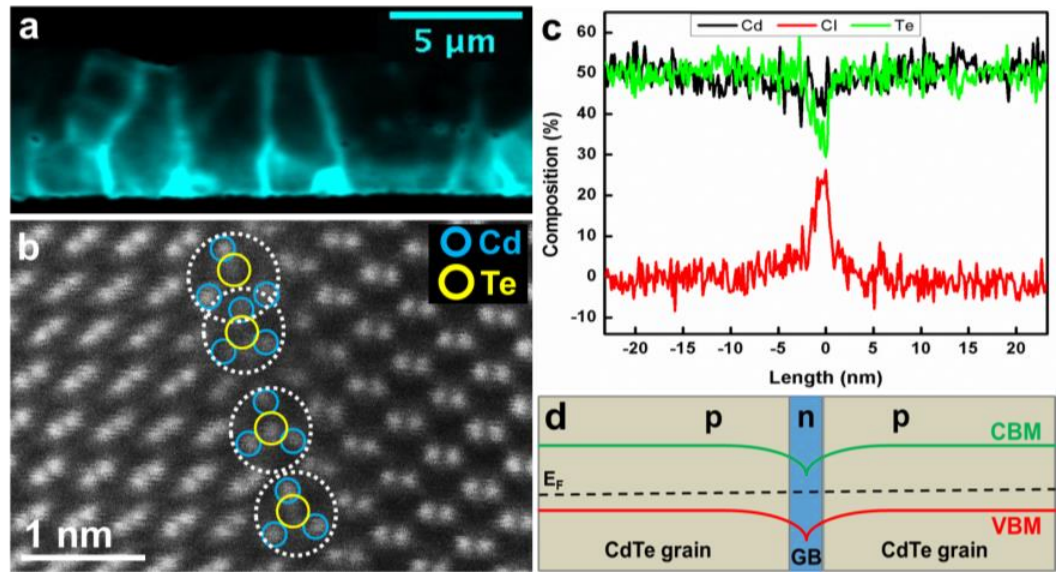

Figure 1. (a) EBIC map shows that the efficiency of GBs in $\mathrm{CdTe}$ cells is higher than grain interiors after $\mathrm{CdCl} 2$ treatment. (b) Z-contrast image shows periodic $\mathrm{Cd} 3 \mathrm{Te}$ dislocation cores along a $\Sigma 9 \mathrm{~GB}$. (c) EELS profiles across the $\Sigma 9$ GB show that nearly a half of the Te atoms have been substituted by $\mathrm{Cl}$ within $\sim 1.5 \mathrm{~nm}$ range at the GB. (d) DFT calculation indicates the GBs have been inverted to n-type by $\mathrm{Cl}$ doping, therefore creating $\mathrm{p}-\mathrm{n}$ junctions between the grain interiors and the GBs. (C. Li et. al. submitted for publication)

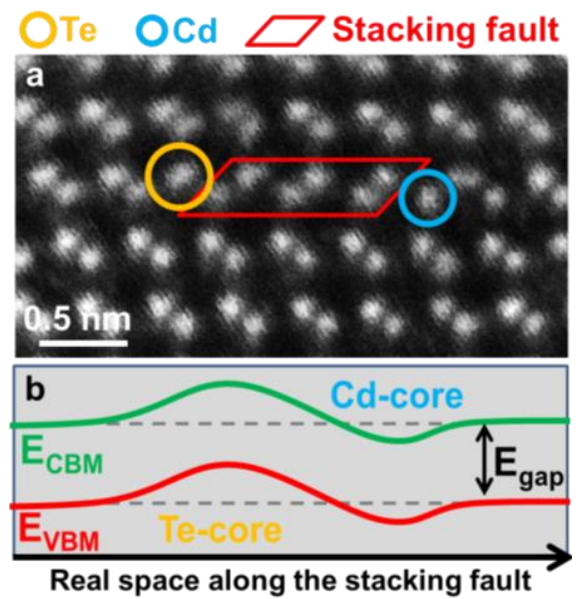

Figure 2. (a) STEM Z-contrast image resolves single $\mathrm{Cd}$ and $\mathrm{Te}$ columns in the Shockley partial dislocation pairs at the ends of an intrinsic stacking fault. (b) DFT calculations indicate that the dislocation pairs induce band bending along the fault, which helps the carrier separation [3].

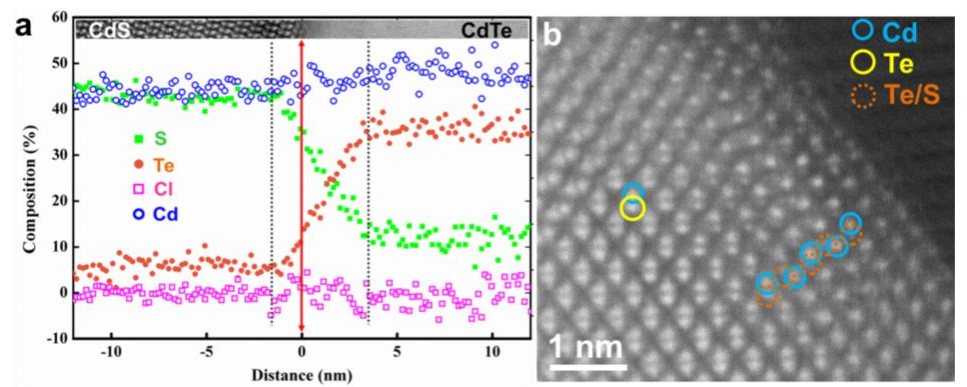

Figure 3. (a) EELS profiles across a $\mathrm{CdTe} / \mathrm{CdS}$ interface show significant Te-S inter-diffusion within $\sim 5 \mathrm{~nm}$ at grain interiors. (b) Z-contrast image directly resolves $\mathrm{S}$ dopants in Te sites. $\mathrm{Cd}-\mathrm{Te} / \mathrm{S}$ dumbbells form wurtzite structure near the interface. 